\title{
Efficacité de la méthode EMDR-sur-le-champ pour le traitement de symptômes comportementaux chez des patients atteints d'une démence sévère
}

\author{
Tamaki Amano \\ École de médecine de l'université de Kyoto, Japon \\ Organisation pour la promotion la recherche sur les troubles développementaux, \\ Maison de repos pour personnes âgées du Japon, Kiyosumien, Japon \\ Motomi Toichi \\ École de médecine de l'université de Kyoto, Japon \\ Organisation pour la promotion la recherche sur les troubles développementaux, Japon
}

\begin{abstract}
Bien que les symptômes principaux de la démence consistent en des déficits neuropsychologiques, affectant particulièrement la mémoire à long terme, la démence comporte souvent des symptômes comportementaux et psychologiques sévères de démence (SCPD). Chez bon nombre de patients, les SCPD ne sont pas traitables par voie médicamenteuse. De tels SCPD partagent souvent certaines caractéristiques avec les symptômes traumatiques et semblent liés au rappel d'événements traumatiques perturbants passés. Comme le protocole EMDR (désensibilisation et retraitement par les mouvements oculaires) standard n'est pas directement applicable aux patients atteints de démence, nous avons développé un protocole modifié : la méthode EMDR-sur-le-champ. Cette étude décrit le protocole et évalue son application à trois patients atteints d'une démence modérée à sévère. Des effets thérapeutiques clairs étaient manifestes et les trois individus ont montré une amélioration marquée de leurs SCPD, avec des résultats maintenus lors du suivi après 6 mois. La pertinence de ces résultats est discutée et des suggestions sont formulées pour la recherche future.
\end{abstract}

Mots-clés : démence ; symptômes comportementaux et psychologiques de la démence (SCPD) ; trauma ; EMDR ; tapotements bilatéraux ; méthode EMDR-sur-le-champ

$\mathbf{L}$ a démence désigne un ensemble de symptômes causés par des troubles affectant le cerveau. Il ne s'agit pas d'une maladie spécifique. Différents troubles peuvent causer la démence, comme la maladie d'Alzheimer (MA) et les accidents cardiovasculaires. Bien que les symptômes principaux de la démence consistent en des déficits neuropsychologiques (tels que l’amnésie, la dyspraxie et la désorientation), la démence implique souvent des problèmes cliniques sévères appelés symptômes comportementaux et psychologiques de la démence (SCPD). Les SCPD sont généralement considérés comme des réactions pathologiques secondaires causées par des changements organiques dans le cerveau ; les symptômes comportementaux comprennent l'agression physique, les hurlements, l'excitation psychomotrice, l'agitation, la déambulation, des conduites culturellement inappropriées, la désinhibition sexuelle, la syllogomanie, les jurons, le shadowing ${ }^{1}$, et les symptômes psychologiques qui comprennent l’anxiété, une humeur dépressive,

This article originally appeared as Amano, T. \& Toichi, M. (2014). Effectiveness of the On-the-Spot-EMDR Method for the Treatment of Behavioral Symptoms in Patients with Severe Dementia. Journal of EMDR Practice and Research, 8(2), 50-65. Translated by Jenny Ann Rydberg. 
des hallucinations et des délires (Lawlor, 2002). Le traitement des SCPD en pharmacothérapie en première option a été tenté (Schmitt, Bernhardt, Moeller \& Heuser, 2004) mais il existe des patients pour lesquels une approche pharmacothérapeutique ne s'avère pas efficace. Il est reconnu qu'il y a des cas dans lesquels la cause des SCPD réside dans les problèmes personnels du patient et on pense que les symptômes pourraient être l'expression de l'état d'esprit particulier du patient, qui aurait endossé les habitudes démentes caractéristiques et qui serait fondé sur le caractère et l'histoire de vie de l'individu (Kitwood, 1993).

Les patients atteints de démence diffèrent en termes des problèmes qu'ils rencontrent habituellement et de la vitesse à laquelle leurs capacités se détériorent. Les caractéristiques de la démence sont communément classées en trois stades : précoce, modérée et tardive. Au stade précoce, les individus retiennent une certaine conscience de leur situation mais deviennent de plus en plus confus et portés à l'oubli. Lors du stade modéré, les symptômes progressent pour se trouver marqués par une perte de la mémoire et une confusion importante, avec un besoin d'assistance quotidienne pour l'habillement, la toilette, la préparation des repas et d'autres tâches. Au dernier stade, les individus sont hautement handicapés et ont besoin d'une prise en charge complète. Ils perdent la capacité à comprendre ou à utiliser la parole ; deviennent incontinents ; ne manifestent aucune reconnaissance de leurs amis ou des membres de leur famille ; ont besoin d'aide pour manger, se laver, prendre leur bain, aller aux toilettes ou s'habiller ; ne reconnaissent plus les objets usuels; et sont perturbés la nuit, agités et agressifs. La perte de mémoire est particulièrement susceptible d'être très prononcée. Les personnes peuvent être incapables de reconnaitre leurs proches ou même leur propre reflet, peuvent croire qu'elles vivent dans un temps appartenant à leur passé, et peuvent être à la recherche de quelqu'un ou de quelque chose de cette époque-là. Elles trouveront probablement difficile de communiquer avec les autres. Elles peuvent perdre graduellement la parole ou elles peuvent répéter quelques mots ou s'écrier de temps en temps.

Les directives de la Société américaine de psychiatrie se concentrent sur les traitements psychothérapeutiques spécifiques suivants pour la MA et la démence : (a) centré sur les comportements, (b) centré sur les émotions, (c) centré sur les cognitions et (d) centré sur la stimulation (American Psychiatric Association, 1999) ; le Comité du groupe pour l'avancement de la psychiatrie en gériatrie (1988) a rapporté qu'une approche accordant une attention particulière aux émotions passées peut être efficace (Bonder, 1994), par exemple, la thérapie par réminiscence (Butler, 1963) et la thérapie par empathie (Jones, 1985). La thérapie par réminiscence est l'une des interventions psychosociales les plus populaires dans les soins aux déments et est hautement appréciée par les équipes et par les patients (Woods, Spector, Jones, Orrell \& Davies, 2009). La thérapie par empathie est une façon d'approcher les adultes aînés avec empathie et compréhension. On pense que les personnes qui ont un âge très avancé peuvent avoir des problèmes non résolus qui dirigent leurs comportements et leurs émotions. Une telle thérapie implique donc simplement la validation des émotions de la personne, en se centrant sur l'aide à la personne pour qu'elle puisse travailler sur les émotions qui sous-tendent sa perte mnésique, sa confusion, sa désorientation et d'autres symptômes de la démence. Les deux thérapies peuvent être efficaces pour la démence légère lors du stade précoce. Cependant, elles ne semblent pas efficaces pour traiter la démence sévère aux stades ultérieurs. La grande majorité des personnes atteintes de démence et de perturbations très prononcées telles que la dépression, l'agressivité et une agitation marquée sont susceptibles de demeurer dans un cadre destiné aux adultes les plus âgés. Le traitement peut impliquer un psychiatre (ou gériatre), une infirmière de coordination, un psychologue qui développe un projet comportemental et une assistante sociale qui intègre la famille au projet de soins. Il est souvent extrêmement difficile de gérer de telles personnes qui manifestent des symptômes SCPD fréquents. Dans notre maison de retraite, nous observons parfois des patients atteints d'une démence sévère, avec des épisodes répétés de hurlements, qui semblent vivre et revivre de manière répétée des souvenirs désagréables (liés à des traumas). Pour de tels patients, les hallucinations peuvent sembler très réelles, comme si quelque chose se passait véritablement à exactement ce moment-là, et ils hurlent fort et demandent de l'aide, injurient ou deviennent violents. De tels comportements se répètent souvent de sorte à devenir des rituels qui partagent certaines caractéristiques avec les symptômes traumatiques en lien avec le rappel d'événements passés et avec la réaction émotionnelle qu'ils suscitent. C'est pourquoi nous considérons que si on regarde au-delà de leurs symptômes de démence, un mécanisme pathologique similaire à l'état de stress post-traumatique (ESPT) pourrait se révéler.

L'EMDR (désensibilisation et retraitement par les mouvements oculaires), un type de psychothérapie développé par Shapiro (1989a, 1989b, 2001), est largement reconnu comme traitement efficace de 
l'ESPT (Bisson \& Andrew, 2007). L’EMDR est décrit comme un bon instrument pour le traitement de jeunes victimes du trauma ; toutefois, peu d'études soutiennent son utilisation chez l'adulte plus âgé (Hyer, 1995 ; Thomas \& Gafner, 1993). Hyer (1995) a décrit le cas du traitement de l'ESPT chez des adultes plus âgés atteints de démence chez qui une amélioration significative a été observée après trois séances d'EMDR ; il a été suggéré que l'EMDR pouvait permettre aux patients de participer au passé comme étant actuellement réel et d'évaluer le déroulement d'un événement dans la perspective de l'observateur. Ces études semblent soutenir l'efficacité de l'EMDR dans le traitement de l'ESPT chez des patients âgés avec une démence très légère. À notre connaissance, il n'y a pas eu de rapports sur l'application de l'EMDR à des patients atteints d'une démence sévère à un stade tardif. Dans cette étude, nous avons tenté l'EMDR sur des patients atteints de démence à un stade tardif. Il existe déjà des protocoles qui sont modifiés pour les enfants (Lovett, 1999) et ceux pour les personnes atteintes de déficits intellectuels (Barol \& Seubert, 2010 ; Mevissen, Lievegoed, Seubert \& Jongh, 2011).

Nous avons employé un protocole similaire dans lequel seuls de simples mots basiques étaient employés dans la communication avec les patients, avec un tapotement sur les deux côtés réalisé en tant que stimulation bilatérale, effectué immédiatement quand l'état agité apparaissait tel que causé par les SCPD, ce que nous appelons la " méthode EMDR-sur-le-champ ». Cette méthode était nécessaire car les personnes atteintes d'une démence sévère ne peuvent pas contrôler leur rappel d'un souvenir traumatique ou communiquer avec un thérapeute dans le cadre de travail d'une séance EMDR. Comme les émotions tendent à apparaitre sous forme de flash-back, nous pratiquons des stimulations bilatérales continues sur-le-champ dans de telles conditions (Amano \& Ichii, 2009). Les trois patients de cette étude étaient des patients atteints d'une démence sévère qui vivaient dans une maison de retraite depuis des années. Ils ont tous montré une amélioration remarquable au niveau des SCPD après les séances d'EMDR.

\section{Méthode}

Les proches ou les tuteurs des patients atteints de démence ont donné leur accord pour la participation, après l'explication des procédures. Un consentement éclairé écrit a été obtenu auprès de tous les proches ou tuteurs. Une approbation éthique a été donnée par le médecin de la maison de retraite.

\section{Patients}

Les patients étaient trois personnes atteintes de démence aux stades modéré ou tardif, à savoir une patiente de 67 ans (MA d'apparition précoce), un patient de 85 ans (démence vasculaire) et un patient de 83 ans (MA). Le premier auteur est une psychologue clinicienne dans une maison de retraite. Tous les patients vivaient dans notre maison de retraite Kiyosumien (centre résidentiel) et étaient pris en charge par des soignants, un médecin spécialisé, une infirmière, une psychologue (le premier auteur), une assistante sociale, et ainsi de suite. Il était souvent extrêmement difficile de gérer les trois patients qui avaient des symptômes SCPD fréquents comme des agressions physiques, des hurlements, de l'excitation psychomotrice, de l'agitation et la déambulation. Comme ils avaient des souvenirs d'événements traumatiques, nous avons soupçonné que leurs SCPD pouvaient comporter des symptômes de type ESPT.

\section{La méthode EMDR-sur-le-champ pour la démence sévère}

Un protocole EMDR standard comprend huit phases (Shapiro, 2001). Cependant, comme la mémoire à long terme des patients à des stades avancés de la démence est défectueuse et que le protocole EMDR standard ne peut pas être appliqué, nous avons développé la méthode EMDR-sur-le-champ pour la démence sévère, dans laquelle les huit phases sont modifiées comme suit. Dans l'EMDR standard, la première phase consiste à obtenir l'histoire du patient, comprenant son aptitude au traitement et les "facteurs de sécurité ». Comme les patients atteints d'une démence sévère ne peuvent pas communiquer leur propre histoire, le thérapeute l'obtient du dossier du patient et de ses proches. La deuxième phase est l'étape de préparation, comprenant la création d'une relation de confiance avec le patient. Comme ces patients ne se rappellent généralement pas leur thérapeute, il importe de mettre en place le traitement à chaque fois comme si le thérapeute rencontrait le patient pour la première fois. La troisième phase EMDR est l'étape d'évaluation qui comprend la sélection du souvenir ciblé et l'identification et l'évaluation de ses composants perceptuels, cognitifs, affectifs et somatiques. Cette phase est abrégée pour l'ajuster aux capacités du patient ou à sa situation urgente, parce qu'il ne peut pas communiquer librement avec nous et se rappeler l'événement traumatique. La méthode EMDR-surle-champ choisit plutôt de considérer que le souvenir traumatique perturbant est révélé par le contenu des symptômes SCPD du patient, car les patients atteints 
d'une démence sévère ne peuvent pas contrôler leurs émotions ou retenir leurs cris. Au cours des quatrième à sixième phases de l'EMDR, le souvenir est retraité à l'aide des stimulations bilatérales. La méthode EMDRsur-le-champ n'utilise que des tapotements comme stimulation bilatérale car la plupart des patients atteints d'une démence avancée ne peuvent contrôler leurs mouvements oculaires en suivant les instructions du thérapeute. La méthode suit une approche modifiée détaillée dans le paragraphe suivant. La septième phase, la clôture de la séance, est également modifiée dans cette méthode. La huitième phase est employée en début de séance et sert à réévaluer les effets de la séance précédente. Comme le patient ne peut pas communiquer, l'efficacité du travail précédent est évaluée en consultant le dossier documentant les symptômes SCPD et en vérifiant auprès des membres de l'équipe le contenu des verbalisations du patient.

Maintenant, nous expliquerons en détail la méthode EMDR sur-le-champ pour la démence sévère. La première étape est la suivante : déterminer si les SCPD correspondent ou non à une reviviscence des expériences du passé, en se basant sur l'observation des comportements du patient. Si votre patient semble revivre l'expérience d'un trauma (en SCPD), suivez les étapes suivantes : (a) positionnez-vous face au patient. Maintenez le contact visuel ; (b) observez l'expression du visage du patient. Parlez au patient en fonction des émotions exprimées sur son visage. En cas d'agitation élevée, parlez aussi calmement que possible, sur un ton restreint et détendu ; (c) employez les mots clés utilisés par le patient, répétez ses mots et prenez des notes. Prêtez attention aux signes non verbaux tels que les expressions du visage décrivant l'inconfort et le désagrément ; (d) pratiquez l'EMDR : centrez-vous sur les souvenirs traumatiques, administrez les stimulations bilatérales à travers les tapotements et installez un sentiment de sécurité avec des mots comme "je suis en sécurité maintenant » ou " c’est fini »; (e) si votre patient est détendu (sorti des SCPD), faites ce qui suit : pratiquez le développement et l'installation de ressources (Korn \& Leeds, 2002) ; cherchez des ressources positives chez le patient, comme des souvenirs ou des sensations (sentiment de voir, de goûter, d'entendre, de humer, de toucher, etc.). Encouragez tout rappel de ressources à travers des mots ou des sensations physiques. Ajoutez des stimulations bilatérales (tapotements) à ces sensations et à ces images.

\section{Exemples de cas}

Dans cette étude, nous avons évalué le traitement de trois patients qui ont bénéficié de la méthode
EMDR-sur-le-champ pour leurs SCPD. Les trois patients, atteints d'une démence modérée à sévère, ont pu suivre le traitement complet avec succès. Une description des trois patients, de leur traitement et des résultats de l'intervention est proposée dans le Tableau 1.

\section{Cas 1}

Tomoko (une femme de 67 ans) était une patiente dont la démence était due à une MA précoce.

Histoire. Des signes d'oubli sont apparus chez Tomoko vers ses 54 ans. La perte de mémoire a progressé graduellement et est devenu évidente vers l'âge de 59 ans. De plus, ont été notées des idées délirantes de s'être fait voler des objets et un déclin au niveau de l'énergie et des activités ; Tomoko a complètement cessé toute activité de ménage ou de cuisine. Les IRM ont révélé une atrophie cérébrale manifeste dans le lobe frontal, dans le lobe pariétal ainsi que dans le lobe temporal intérieur. Les résultats de la TEP ont également indiqué une forte possibilité de MA. À la suite d'un examen complet, la patiente a été diagnostiquée comme " un cas assez spécial de MA avec une apparition relativement précoce et une atteinte de la cognition visuelle et de la compréhension ».

À l'âge de 60 ans, quand la MA était estimée à un stade sévère, le médicament pour le traitement de la démence (donépézil) a été prescrit. Toutefois, comme la patiente est devenue agitée et agressive suite à la prise de ce médicament, le traitement a été interrompu à la demande de sa famille. Les résultats principaux d'un examen neuropsychologique dans un hôpital étaient les suivants : Mini-Mental State Examination (MMSE) 15/30 et Wechsler Adult Intelligence ScaleRevised (WAIS-R) : QI verbal (QIV) 69, QI performance (QIP) 50, QI total (QIT) 59. La patiente a eu régulièrement recours aux services d'hôpital de jour d'une maison de retraite à partir de ses 60 ans. En plus de la progression de la démence, des symptômes tels que la déambulation, des paroles injurieuses et des violences contre les membres de l'équipe se sont intensifiés. Aussi, de manière caractéristique de la MA, Tomoko avait des hallucinations visuelles et auditives, se parlait à elle-même et manifestait fréquemment des conduites agitées telles que des cris.

À l'âge de 67 ans, le score MMSE était 0/30. Sur la base de ces résultats, Tomoko a reçu un diagnostic de démence sévère avec des SCPD prononcés.

Son histoire de vie, telle que décrite par son mari, était que Tomoko a dû connaître la pauvreté pendant son enfance. Ses parents, sa fratrie et ses proches ont été presque totalement absents lors de son mariage. Après le mariage, Tomoko a vécu sous l'autorité d'une 
TABLEAU 1. Traitement EMDR de trois patients atteints de démence avec la méthode sur-le-champ

\begin{tabular}{|c|c|c|c|c|c|c|}
\hline Cas & $\begin{array}{l}\text { Niveau de } \\
\text { démence } \\
\text { (MMSE) }\end{array}$ & Maladies & $\begin{array}{l}\text { Symptômes } \\
\text { comportementaux et } \\
\text { psychologiques de la } \\
\text { démence (SCPD) }\end{array}$ & $\begin{array}{l}\text { Événements de } \\
\text { vie désagréables }\end{array}$ & $\begin{array}{l}\text { Nombre de } \\
\text { séances }\end{array}$ & Résultats \\
\hline $\begin{array}{l}\text { Tomoko, } \\
\text { femme } \\
\text { de } 67 \text { ans }\end{array}$ & $\begin{array}{l}\text { Sévère } \\
\qquad(0 / 30)\end{array}$ & $\begin{array}{l}\text { Alzheimer } \\
\text { précoce }\end{array}$ & $\begin{array}{l}\text { Cris, violences } \\
\text { verbales }\end{array}$ & $\begin{array}{l}\text { Violences des } \\
\text { créanciers dans } \\
\text { l'enfance }\end{array}$ & Six & $\begin{array}{l}\text { Railleries chro- } \\
\text { niques et injures } \\
\text { réduites. } \\
\text { La déambulation } \\
\text { se produisait } \\
\text { toujours fré- } \\
\text { quemment } \\
\text { en chantant } \\
\text { ses chansons } \\
\text { préférées ou en } \\
\text { silence. }\end{array}$ \\
\hline $\begin{array}{l}\text { Taro } \\
\qquad \text { (homme } \\
\text { de } 85 \text { ans) }\end{array}$ & $\begin{array}{r}\text { Modérée } \\
(7 / 30)\end{array}$ & $\begin{array}{l}\text { Maladie } \\
\text { cérébro- } \\
\text { vasculaire }\end{array}$ & $\begin{array}{l}\text { Excitation } \\
\text { psychomotrice, } \\
\text { accompagnée } \\
\text { de cris, de } \\
\text { déambulation, de } \\
\text { perturbations du } \\
\text { sommeil, de délires } \\
\text { nocturnes, de } \\
\text { résistance contre } \\
\text { les soignants. }\end{array}$ & $\begin{array}{l}\text { 1: L’incendie } \\
\text { 2: Le cambriolage }\end{array}$ & Quatre & $\begin{array}{l}\text { Diminution de } \\
\text { l'excitation } \\
\text { psychomotrice } \\
\text { accompagnée de } \\
\text { cris soudains qui } \\
\text { se poursuivait } \\
\text { habituellement } \\
\text { pendant des } \\
\text { heures }\end{array}$ \\
\hline $\begin{array}{l}\text { Toshio } \\
\qquad \text { (homme } \\
\text { de } 83 \text { ans) }\end{array}$ & $\begin{array}{l}\text { Sévère } \\
\qquad(0 / 30)\end{array}$ & $\begin{array}{l}\text { Maladie } \\
\text { d'Alzheimer }\end{array}$ & $\begin{array}{l}\text { Déambulation, } \\
\text { cris, excitation } \\
\text { psychomotrice, } \\
\text { habitudes } \\
\text { alimentaires } \\
\text { bizarres }\end{array}$ & $\begin{array}{l}\text { 1: un événement } \\
\text { non défini à } \\
\text { Yokosuka } \\
\text { 2: en colère } \\
\text { concernant } \\
\text { quelque chose } \\
\text { que le patient a } \\
\text { crié de manière } \\
\text { répétée }\end{array}$ & Cinq & $\begin{array}{l}\text { S'est calmé et a } \\
\text { souri d'avantage } \\
\text { après l'EMDR }\end{array}$ \\
\hline
\end{tabular}

Note. MMSE $=$ Mini-Mental State Examination

belle-mère très exigeante. Avec son mari, ils ont tenu un magasin de friandises à l'ancienne et ont élevé quatre enfants : deux fils et deux filles. Tomoko subissait quotidiennement les violences verbales de sa belle-mère ; elle était une bru soumise et obéissante. Il y avait également plusieurs salariés auxquels elle ne répondait jamais. Tomoko avait décrit au premier auteur une expérience douloureuse où des créanciers sont entrés dans son foyer d'enfance pour enlever des objets.

Le principal SCPD de Tomoko était un état de déambulation. Les dossiers de la maison de retraite indiquent :

Déambule seule à travers les couloirs avec une posture penchée vers l'avant, tout en poussant des cris forts. Quand un soignant tente de l'arrê- ter, elle le raille et le hue, et tente de se défaire des mains du soignant pour continuer à déambuler. Le contenu de ses railleries est presque toujours identique : «trois personnes. . trois personnes, et une personne de plus, ça fait quatre personnes. .. ». Elle a toujours une hallucination impliquant trois personnes en lien avec la scène traumatique.

Évaluation. Comme la déambulation était considérée comme caractéristique de ses SCPD, elle a été choisie comme cible pour les dossiers. L'analyse comportementale de la déambulation (méthode d'échantillonnage temporel) était réalisée avant le traitement, avec des données enregistrées toutes les 30 minutes au cours d'une période quotidienne d'échantillonnage pendant 


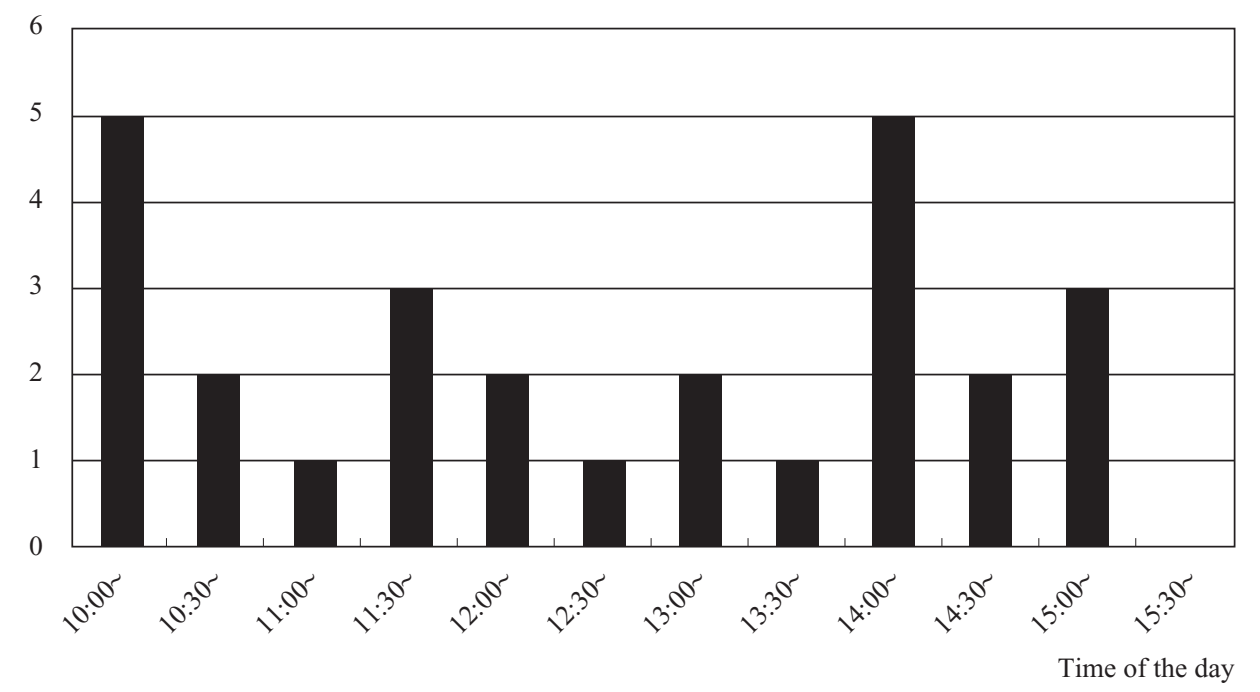

FIGURE 1. Fréquence quotidienne de la déambulation du Cas 1 avant traitement.

Note. Number of times wandering took place $=$ nombre de fois où la déambulation a eu lieu ; Time of the day $=$ Heure de la journée.

cinq jours. Les résultats apparaissent dans la Figure 1. Le nombre de fois où la patiente a déambulé au cours des cinq jours est illustré sur l'axe vertical et l'heure de la journée sur l'axe horizontal. Comme illustré par le graphique, les périodes associées à une déambulation élevée se situent vers 10h00-11h00, 11h30-12h00 et 14h00-16h00. Un extrait du dossier documentant les comportements de la patiente pendant la période d'intervention apparaît dans le Tableau 2. Le premier auteur a continué à contacter les soignants pour évaluer le niveau de SCPD manifestés par la patiente plus de 6 mois après l'intervention EMDR.

Traitement. L'intervention a été appliquée à six occasions sur une période de trois mois par le premier auteur dans la salle de jour de la maison de retraite médicalisée. Comme illustré dans la Figure 1, les moments correspondant à une fréquence élevée de déambulation se situaient entre 10 heures et 11 heures ainsi qu'entre 14 heures et 16 heures. Quand Tomoko manifestait le comportement problématique ciblé - des hurlements, des violences verbales et des violences physiques au cours d'hallucinations et de délires - la méthode EMDR-sur-le-champ était réalisée. Un résumé de la scène traumatique est la suivante:

Tout d'un coup, trois agents de recouvrement féminins entrent dans la maison pour recouvrer les dettes du père de la patiente. Une quatrième personne, un homme, arrive également. Ils prennent tout ; ils forcent même la petite fille
Tomoko à enlever ses vêtements et ils les emportent. Son père est sous le même toit, mais Tomoko se cache dans la chambre voisine.

Tomoko, qui déambulait tout en hurlant fortement et en lançant des injures dans un état agité, s'est laissé guider calmement vers un canapé dans un coin tranquille de la salle de séjour. On l'a fait s'asseoir et le premier auteur s'est assis directement en face d'elle pour mener la séance avec des tapotements sur ses genoux. (Note : en comparaison de l'EMDR standard, l'évaluation a été fortement raccourcie. Les modifications étaient opérées en fonction des capacités et de la situation urgente de la patiente.) Des séances EMDR ont été effectuées. Dès que la patient devenait agitée en raison de l'hallucination des agents de recouvrement, la stabilisation était obtenue en lui disant « c'est fini ; les personnes sont parties et elles ne reviendront plus jamais ; tout va bien » et " votre mari vous protège ", en administrant des tapotements bilatéraux. De plus, lors d'une réunion sur ce cas, tous les membres de l'équipe ont été invités à créer le contact visuel avec Tomoko et à lui dire une phrase clé, "c'est fini ; tout va bien " tout en apportant des tapotements bilatéraux dès qu'elle devenait agitée. Selon le dossier, les périodes d'évaluation initiale et d'intervention se sont étendues sur deux mois environ. Afin d'investiguer les effets des interventions, les soignants ont reproduit des extraits des relevés quotidiens pour Tomoko dans un tableau (voir Tableau 2). 
Histoire. Taro a reçu le diagnostic de DVa causée par les séquelles d'un accident vasculaire cérébral lorsqu'il avait 79 ans. En raison de sa paraplégie, il fut admis en maison de retraite médicalisée. On lui a prescrit du tiapride, un médicament destiné à améliorer le débit sanguin cérébral et le métabolisme cérébral du glucose. Les symptômes de SCPD incluaient les déplacements incessants en fauteuil roulant, des perturbations du sommeil, des délires nocturnes, la résistance contre les soignants et des hurlements. Ils se poursuivaient pendant toute la journée. À l'âge de 85 ans, le MMSE indiquait une démence modérée avec un score de $7 / 30$. Un test évaluant la dépression chez la personne âgée produisait toutefois un résultat normal. L'inventaire d'agitation de Cohen-Mansfield (CMAI ; Cohen-Mansfield, 1986), rempli par un soignant, donnait un score élevé de 51 ; les items particulièrement notables étaient " la déambulation », « les hurlements ", "le fait d'appeler souvent un soignant » et « l'excitation psychomotrice ». Ceux-ci se produisaient plusieurs fois par heure. Ces tests reflètent une démence modérée avec des SCPD évidents.

Les membres de sa famille racontaient que Taro avait fait la guerre et se trouvait en Mandchourie (Chine) à la fin de la deuxième guerre mondiale en 1945. De retour au Japon, après le long éloignement de la guerre, il a d'abord travaillé dans une entreprise puis comme concierge dans une école. Sa maison fut démolie dans un incendie et fut aussi la cible d'un cambriolage. Le journal du soignant indiquait que sa vie dans la maison de retraite était généralement calme et tranquille, mais que quand il fermait les yeux et basculait dans un état délirant, cet état se poursuivait pendant plus d’une heure; il hurlait comme s'il avait mal et criait à l'aide. Dans le dossier du soignant, les informations suivantes étaient notées concernant le patient :

Quand on le laisse seul, Taro devient nerveux. Parfois il se parle à lui-même, les yeux fermés, puis survient un changement soudain, tel qu'un épisode de hurlements. Il semble très mal à l'aise dans ces situations et on dirait qu'il va pleurer. Les délires et les hallucinations sont fréquents. Quand on lui parle, l'agitation diminue et il se calme. Quand il est de bonne humeur, il parle de son enfance ou d'autres patients. Il participe activement à la musicothérapie qu'il semble apprécier.

Évaluation. Pour obtenir des données destinées à l'analyse comportementale des SCPD de Taro, des renseignements étaient notés toutes les 30 minutes pendant 24 heures au cours de dix jours. Les comportements ciblés étaient une " excitation psychomotrice accompagnée de hurlements » et des « mots prononcés dans un état d'excitation psychomotrice ». En indiquant les résultats de l'analyse comportementale, la Figure 2

\footnotetext{
Number of times

when

restlessness

occurred
}

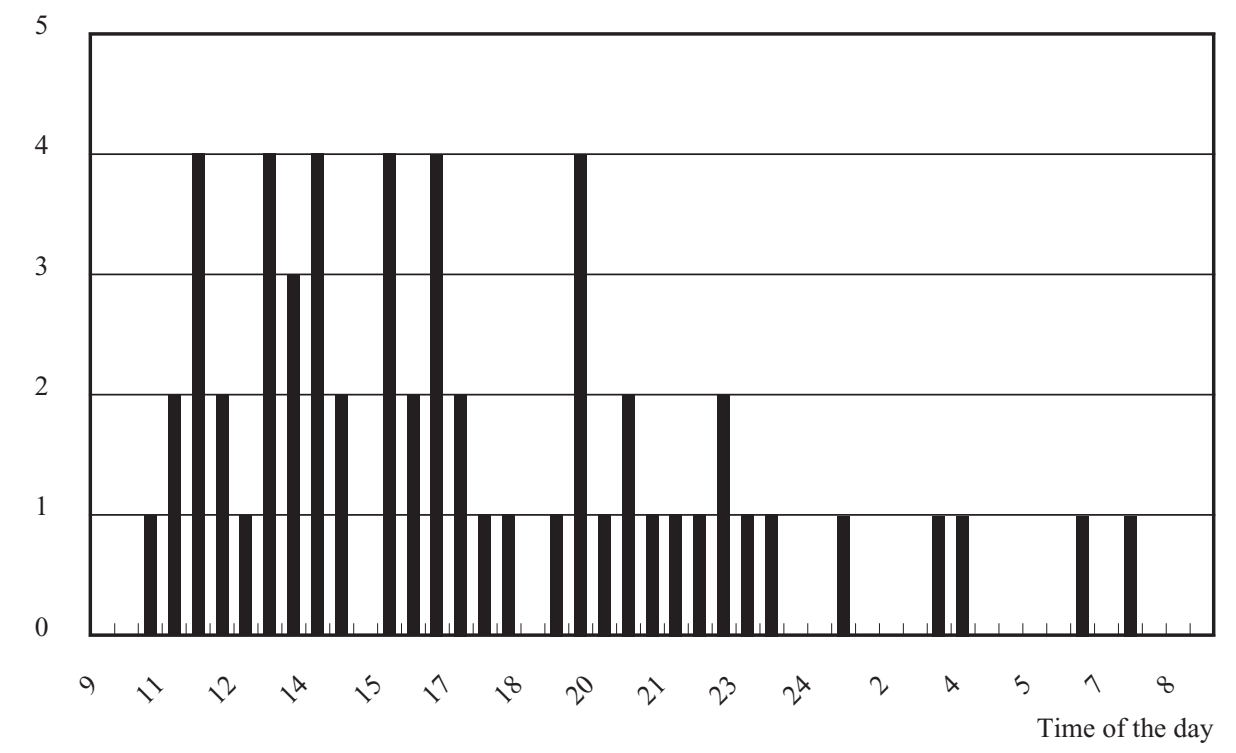

FIGURE 2. Fréquence de l'excitation psychomotrice du Cas 2 pendant une journée entière Note. Number of times when restlessness occurred = nombre de fois où a eu lieu l'excitation psychomotrice ; Time of the day $=$ heure de la journée. 


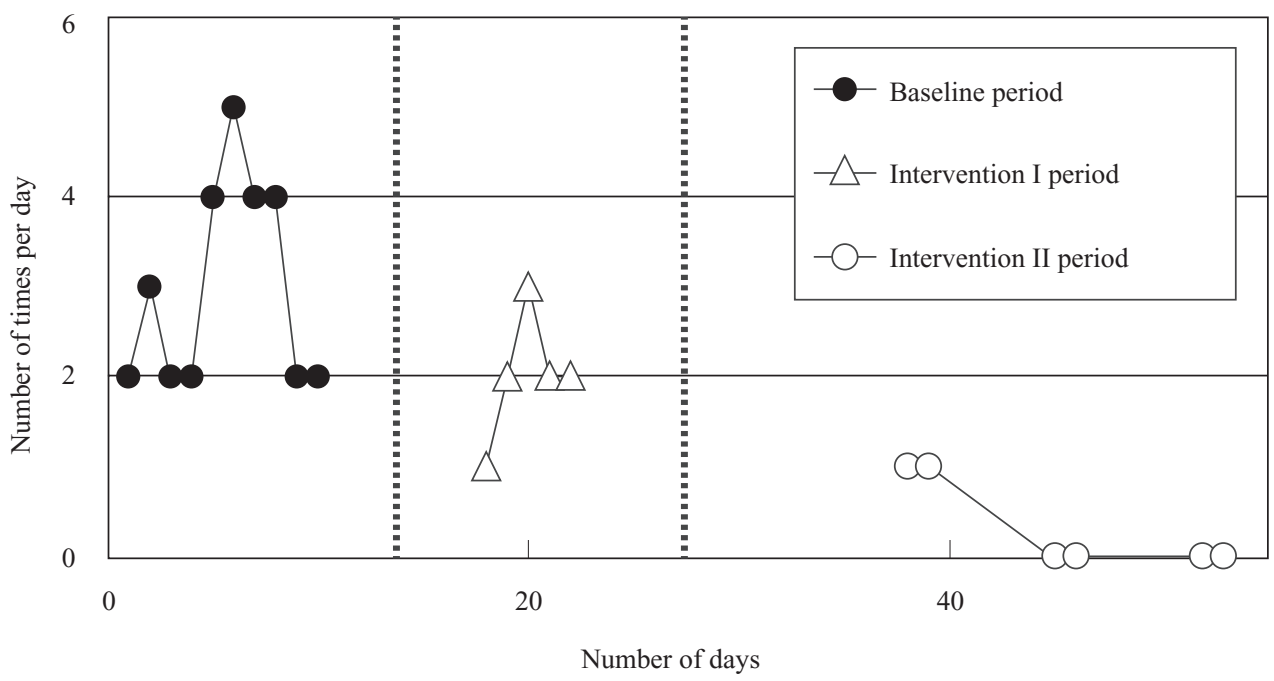

FIGURE 3. Changement au cours du temps du nombre de fois où a eu lieu "l'excitation psychomotrice accompagnée de hurlements » chez le Cas 2

Note. Number of times per day $=$ nombre de fois par jour ; Number of days $=$ nombre de jours ; Baseline period $=$ période d'évaluation initiale ; Intervention I period = période d'intervention I ; Intervention II period = période d'intervention II.

montre la fréquence des moments d'excitation psychomotrice. L'axe vertical montre le nombre total de fois où l'excitation psychomotrice a eu lieu tandis que l'axe horizontal indique l'heure de la journée. Comme le montre le graphique, l'excitation psychomotrice était beaucoup plus fréquente en journée et les périodes avec la plus grande fréquence était $11 \mathrm{~h} 00-11 \mathrm{~h} 30,13 \mathrm{~h} 30$ 15h00, 16h00-17h00 et 19h30-20h00. Ceci indique que l'excitation psychomotrice était la plus fréquente pendant les moments non structurés en journée et juste avant le coucher, lorsque le patient était seul. Ces comportements étaient peu fréquents pendant les repas et collations.

Traitement. L'intervention était administrée dans la salle de séjour de la maison de retraite médicalisé pendant trois mois. Les résultats se trouvent dans la Figure 3. Nous avons appliqué deux types d'intervention : l'intervention I impliquait seulement qu'un soignant parle au patient (conversation), tandis que l'intervention II était une séance d'EMDR avec le premier auteur. Les comportements problématiques ciblés étaient l'excitation psychomotrice accompagnée de hurlements. Dans l'intervention I, quand Taro montrait des signes annonciateurs d'un état d'excitation psychomotrice, un soignant faisait une intervention de contrôle du délire et s'adressait à Taro avec des mots apaisants comme "Ouvrez les yeux s'il vous plaît. Vous êtes en sécurité ici. Tout va bien. » Ce type d'intervention de la part des soignants s'est déroulé pendant cinq jours. Dans l'intervention II, le Taro recevait une séance EMDR avec le premier auteur ; dès qu'il semblait revivre la scène de l'incendie ou du cambriolage et exprimer ces scènes à travers ses hurlements, une stimulation bilatérale était appliquée. La première scène traumatique était l'incendie. Sa maison a pris feu à minuit. Il voulait sortir de la maison mais il ne parvenait pas à déverrouiller la porte. Sa famille et lui étaient piégés par les flammes, mais tous ont été sauvés de la maison en feu par les pompiers. La seconde scène traumatique était le cambriolage. Taro a trouvé un intrus chez lui et a crié. Le cambrioleur a tout d'un coup frappé Taro au visage. Quand un policier est arrivé, le cambrioleur a été arrêté. Les deux événements traumatiques ont été approchés avec la même méthode EMDR-sur-le-champ. Quand Taro s'agitait, hurlant en lien avec l'incendie (ou le cambriolage), les yeux fermés, la thérapeute se plaçait devant lui et continuait à appliquer des tapotements bilatéraux en parlant de la scène. Comme ses récits traumatiques progressaient rapidement avec les tapotements, il pouvait atteindre la fin de l'événement traumatique. En ouvrant ses yeux à la fin de l'histoire traumatique, il se rendait compte que l'événement était passé. Comme son fils était policier, il était convaincu que son fils pouvait le protéger.

Le Tableau 3 montre des extraits du dossier patient tenu par les soignants pour la période d'évaluation initiale et les périodes d'intervention I et II. Au cours de la période d'évaluation initiale, les états d'excitation psychomotrice de Taro duraient chaque fois plus d'une heure. Le patient avait l'air anxieux et semblait avoir mal, tandis que ses paroles exprimaient de la peur. Pendant la période d’intervention I, les 
TABLEAU 3. Extraits du dossier patient du Cas 2 tenu par les soignants

\begin{tabular}{|c|c|c|c|}
\hline Période & Jour/mois & Heure & Phrases criées \\
\hline \multirow[t]{30}{*}{ Évaluation initiale } & \multirow[t]{2}{*}{$25 / 6$} & $12 \mathrm{~h} 30$ & J'ai la tête qui tourne! \\
\hline & & $14 \mathrm{~h} 10$ & $\begin{array}{l}\text { Quelqu'un arrive en se dépêchant ! Ma grande sœur s'est-elle } \\
\text { effondrée? }\end{array}$ \\
\hline & \multirow[t]{3}{*}{$26 / 6$} & $6 \mathrm{~h} 00$ & Monsieur! (crie) \\
\hline & & $18 \mathrm{~h} 15$ & Ohhh !.....oh ! (crie) \\
\hline & & $19 \mathrm{~h} 00$ & Ohhh ! (crie) \\
\hline & \multirow[t]{4}{*}{$27 / 6$} & $07 \mathrm{~h} 00$ & Ahhh !..... ah ! (crie) \\
\hline & & 9 h00 & Je ne peux pas voir ces lettres (crie, se parle à lui-même \\
\hline & & $11 \mathrm{~h} 00$ & $\begin{array}{l}\text { Pourquoi des choses aussi terribles m'arrivent toujours ? Comme } \\
\text { ces voleurs... (quand on lui parle, essaie de frapper comme pour } \\
\text { se défendre) }\end{array}$ \\
\hline & & $20 \mathrm{~h} 00$ & J'ai perdu mon argent, mes 20000 yen ! J'ai fait une chose terrible ! \\
\hline & \multirow[t]{4}{*}{$28 / 6$} & $11 \mathrm{~h} 30$ & Ohhh !.....oh ! (crie) \\
\hline & & $12 \mathrm{~h} 20$ & Ohhh !.....oh ! (crie) \\
\hline & & $13 \mathrm{~h} 00$ & Ohhh !.....oh ! (crie) \\
\hline & & $21 \mathrm{~h} 00$ & Ohhh !.....oh ! (crie) \\
\hline & \multirow[t]{5}{*}{$29 / 6$} & $4 \mathrm{~h} 00$ & Où est.... ? (crie) \\
\hline & & $4 \mathrm{~h} 30$ & Je dois aller à... \\
\hline & & $11 \mathrm{~h} 30$ & Ohhh !.....oh ! (crie) \\
\hline & & $14 \mathrm{~h} 00$ & Ohhh !.....oh ! Il n’y a personne (crie) \\
\hline & & $19 \mathrm{~h} 00$ & Ohhh !.....oh ! (crie) \\
\hline & \multirow[t]{4}{*}{$30 / 6$} & 06h00 & Ohhh !.....oh ! (crie) \\
\hline & & $09 \mathrm{~h} 00$ & Ahhh !......ah! \\
\hline & & $11 \mathrm{~h} 30$ & Où est...? \\
\hline & & $13 \mathrm{~h} 30$ & Où est...? \\
\hline & \multirow[t]{4}{*}{$1 / 7$} & $9 \mathrm{~h} 30$ & Dis le moi! \\
\hline & & $15 \mathrm{~h} 30$ & Ohhh !.....oh ! (crie) \\
\hline & & $17 \mathrm{~h} 30$ & Ohhh !.....oh ! Il y a le feu et le criminel s’est enfoui ! J’ai été brûlé ! \\
\hline & & $23 \mathrm{~h} 30$ & Ohhh !.....oh ! J’ai un pneu crevé ! Un pneu crevé ! \\
\hline & \multirow[t]{2}{*}{$2 / 7$} & $9 \mathrm{~h} 30$ & Ohhh !.....oh ! (crie) \\
\hline & & $13 \mathrm{~h} 30$ & S'iiiiil vou-ous plaîî̀t ! \\
\hline & \multirow[t]{2}{*}{$3 / 7$} & $13 \mathrm{~h} 30$ & J’aurais dû aller travailler aujourd'hui ! J’ai oublié! \\
\hline & & $16 \mathrm{~h} 30$ & S’iiiiil vou-ous plaînît! \\
\hline \multirow{10}{*}{$\begin{array}{l}\text { Période d'intervention I } \\
\text { (un soignant lui parle) }\end{array}$} & $11 / 7$ & $10 \mathrm{~h} 30$ & Ahhh !..... ah ! Maintenant je peux plus rien faire \\
\hline & \multirow[t]{2}{*}{$12 / 7$} & $13 \mathrm{~h} 30$ & Ahhh !....ah !... Maintenant j’ai plus besoin de rien \\
\hline & & $1 \mathrm{~h} 00$ & Ohhh $! \ldots$ oh $! . .$. Juste une fois. \\
\hline & \multirow[t]{3}{*}{$13 / 7$} & $15 \mathrm{~h} 30$ & Ahhh ! Ahhh ! La porte est fermée ! \\
\hline & & $22 \mathrm{~h} 00$ & Ohhh !.....oh ! (crie) \\
\hline & & $2 \mathrm{~h} 50$ & Il y a un... (agité) \\
\hline & \multirow[t]{2}{*}{$14 / 7$} & $13 \mathrm{~h} 30$ & Ohhh !.....oh ! (crie) \\
\hline & & $16 \mathrm{~h} 30$ & Euhhh !.....euh ! (crie) \\
\hline & \multirow[t]{2}{*}{$15 / 7$} & $13 \mathrm{~h} 30$ & Euhhh !.....euh ! (crie) \\
\hline & & $16 \mathrm{~h} 30$ & Vite ! Vite ! (crie) \\
\hline
\end{tabular}


TABLEAU 3. Extraits du dossier patient du Cas 2 tenu par les soignants (Continued)

\begin{tabular}{|c|c|c|c|}
\hline Période & Jour/mois & Heure & Phrases criées \\
\hline \multirow{7}{*}{$\begin{array}{l}\text { Période d'intervention II } \\
\text { (EMDR) }\end{array}$} & $1 / 8$ & oh00 & Ohhh !.....oh ! (crie) (reçoit une tasse de thé mais ne se calme pas) \\
\hline & & oh30 & (Se calme et dort jusqu'au matin) \\
\hline & $2 / 8$ & $12 \mathrm{~h} 30$ & $\begin{array}{l}\text { (Au déjeuner, comportements comme éparpiller son riz et les } \\
\text { autres aliments sur son assiette et sortir les fleurs du vase sur sa } \\
\text { table. Ne parle pas fort.) }\end{array}$ \\
\hline & $8 / 8$ & - & $\begin{array}{l}\text { Garde les yeux fermés et reste silencieux. Même quand on lui } \\
\text { parle, reste silencieux. L'expression du visage ne reflète pas } \\
\text { de douleur ni d'anxiété. }\end{array}$ \\
\hline & $9 / 8$ & - & $\begin{array}{l}\text { Garde les yeux fermés et reste silencieux. } \\
\text { Quand on lui parle, ouvre les yeux. }\end{array}$ \\
\hline & $15 / 8$ & - & $\begin{array}{l}\text { Garde les yeux fermés et reste silencieux. } \\
\text { Se déplace en fauteuil roulant, seul. }\end{array}$ \\
\hline & $17 / 8$ & - & $\begin{array}{l}\text { Ne répond pas et n'ouvre pas les yeux quand on lui parle. Ne parle } \\
\text { pas fort. }\end{array}$ \\
\hline
\end{tabular}

soignants lui parlaient calmement. La fréquence des hurlements et la durée des états d'excitation psychomotrice ont diminué légèrement, mais les hurlements indiquant une peur de l'incendie et du cambriolage n'ont pas cessé. De plus, il n'y avait presqu'aucun changement au niveau du contenu des hurlements. Après la période d'intervention II, au cours de laquelle des séances EMDR ont été réalisées, le nombre de fois où Taro basculait dans un état d'excitation psychomotrice s'est considérablement réduit et les hurlements ont cessé presqu'entièrement. En particulier, après ces séances, le dossier ne relève aucune mention de l'incendie ou du cambriolage. Taro passait encore de longs moments les yeux fermés en journée. Il dormait bien la nuit et ses hurlements nocturnes ont presque disparu. Toutefois, son excitation psychomotrice ne s'est pas complètement arrêtée.

La Figure 3 montre le changement au niveau du " nombre de fois qu'a eu lieu une excitation psychomotrice accompagnée de hurlements » au cours du temps. L'axe vertical indique le nombre de fois où s'est produite "l'excitation psychomotrice accompagnée de hurlements ", tandis que l'axe horizontal montre les jours auxquels les notes ont été prises. Le graphique indique que l'excitation psychomotrice accompagnée de hurlements se produisait en moyenne trois fois par jour, presque tous les jours pendant la période initiale d'évaluation. Pendant l'intervention I, quand les soignants parlaient au patient, le nombre moyen de fois s'est réduit à deux environ. Enfin, l'intervention II avec des séances EMDR avec la méthode sur-le-champ a produit une diminution. De plus, après le traitement du souvenir traumatique pendant le déroulement des séances EMDR, le nombre d'épisodes de hurlements s'est réduit à zéro.

Résultats. Taro souffrait depuis des années de symptômes qui semblaient correspondre à des reviviscences de scènes traumatiques impliquant un incendie et un cambriolage. Quelques séances EMDR ont conduit à une diminution nette de l'excitation psychomotrice accompagnée de hurlements soudains qui perduraient généralement pendant des heures. Même si après les séances EMDR il continuait à se déplacer pendant des heures en fauteuil roulant, les yeux fermés, il n'a quasi plus basculé dans un état d'esprit douloureux se prolongeant plusieurs heures d'affilée. Environ six mois après l'intervention EMDR, ce patient est décédé à la suite d'une maladie chronique - l'asthme. Cependant, au cours des six derniers mois de sa vie, il n'y avait plus aucun symptôme de reviviscence des scènes de l'incendie et du cambriolage.

\section{Cas 3}

Toshio (un homme de 83 ans) était un patient atteint d'une démence sévère en lien avec la maladie d'Alzheimer.

Histoire. Toshio a commencé à perdre la mémoire vers l'âge de 70 ans. Vers l'âge de 75 ans, les symptômes avaient connu une progression considérable au point où il avait du mal à s'habiller et à se déshabiller. Le diagnostic de démence sévère causée par la maladie d'Alzheimer était fondé sur une IRM dans le service de chirurgie cérébrale. Les SCPD comprenaient des symptômes comme la déambulation, des 
perturbations du sommeil se traduisant par une inversion jour-nuit, des délires ainsi que des cris toute la journée. À l'âge de 83 ans, le résultat du MMSE était de $0 / 30$, reflétant une démence très avancée et des mesures de fonctionnement détérioré telles qu'une incapacité totale à communiquer. Le score CMAI, évalué par un soignant, était élevé à 82 . Les symptômes marquants étaient "la déambulation ", " les hurlements ", " appeler souvent les soignants " et " l'excitation psychomotrice ", qui se produisaient plusieurs fois par heure, ainsi que « des habitudes alimentaires bizarres " et " habillage et déshabillage inappropriés ", qui se produisaient plusieurs fois par jour. On le considérait comme un patient atteint d'une démence sévère avec des SCPD évidents.

Ses proches nous ont parlé de son histoire.

Toshio était un officier de l'armée qui avait vécu à Yokosuka (une ville avec une base militaire au Japon). Toshio boitait, ayant été blessé aux jambes pendant la deuxième guerre mondiale. Après la guerre, il a travaillé dans une entreprise.

Sa vie quotidienne dans la maison de retraite se déroulait comme suit (à partir du journal du soignant) :

Toshio essaie souvent de se lever et de quitter son fauteuil roulant. Comme sa démarche est très instable et que le risque de chute est élevé, un soignant doit être présent et disponible en tout temps. Pendant les repas, il attrape les aliments avec ses mains pour tenter de se nourrir. Il manifeste aussi des troubles des conduites alimentaires comme le fait de mordre et de mâchonner une tasse, sa brosse à dents ou les boutons de ses habits. Il pousse parfois des cris forts et soudains, frappe les personnes qui l'entourent et émet des sons bizarres. La nuit, son sommeil tend à être perturbé et il lui arrive fréquemment de se lever toutes les heures ou toutes les deux heures.

Il a été diagnostiqué comme un patient atteint d'une démence sévère avec des SCPD sévères. En général, un traitement pharmacologique s'imposerait dans de tels cas, mais il avait des antécédents de diabète, d'hypertension et d'hyperlipidémie qui l'empêchaient de prendre des médicaments puissants.

Traitement. L'intervention a eu lieu à la maison de retraite médicalisée pendant trois mois. Deux types d'intervention ont été utilisés : l'intervention I impliquait qu'un soignant parle au patient (conversation), tandis que l'intervention II impliquait un traitement pharmacologique et des séances EMDR. Pendant la période d'intervention I, le patient était étroitement surveillé et pris en charge par un soignant. Comme Toshio se trouvait constamment dans un état d'excitation psychomotrice, un soignant lui était spécialement dédié pour lui apporter une prise en charge individuelle. Pendant la période d'intervention II, un traitement pharmacologique était administré trois fois par jour, après chaque repas, à savoir un extrait de Yokukansan en granulés (un complément alimentaire chinois, une phytothérapie considérée comme un tranquillisant léger) et deux sortes d'inducteur du sommeil. La séance d'EMDR se centrait sur deux scènes traumatiques cibles. Dans la première scène : tout élément de Yokosuka, quand Toshio semblait voir quelque chose avec un regard porté vers le haut de manière oblique. Il criait : "Yokosuka, à Yokosuka (le nom d'une base militaire) ". La deuxième scène impliquait le fait d'être en colère contre quelque chose, avec des cris très forts, répétés : «J’ai pas besoin de ça! Ça marche pas comme ça! Ça sert à rien ! J'en veux pas! » Il semblait très énervé. Pendant ces deux sortes d'excitation psychomotrice, comme il était peu susceptible de réagir, le traitement des séances EMDR a été administré à travers la méthode sur-le-champ.

Le Tableau 4 montre le dossier de la période d'évaluation initiale et des périodes des interventions I et II, avec des extraits des notes des soignants. Pendant la période d'évaluation initiale, Toshio se trouvait dans un état d'excitation psychomotrice et se trouvait souvent incapable de dormir la nuit, criant constamment. Quand il faisait des cauchemars ou était fâché, il se levait tout d'un coup. C'est pourquoi, compte tenu du risque de chute, un soignant particulier lui a été affecté. Dans l'intervention I, un soignant parlait calmement à Toshio. Le nombre de fois où il a crié ou la durée des états d'excitation psychomotrice a baissé légèrement. Il était nécessaire, toutefois, de lui apporter une prise en charge individuelle continue en raison de son excitation psychomotrice et du risque continu de chute. Pendant l'intervention II, après les séances EMDR, l'excitation psychomotrice causée par les hallucinations s'est considérablement réduite. En même temps, les hurlements ont presque cessé également. Il s'est calmé à un degré suffisant pour qu'une prise en charge individuelle ne soit plus nécessaire. Lorsqu'il semblait devenir trop excité, des tapotements bilatéraux lents étaient administrés par le soignant. Cela le calmait.

Résultats. Toshio avait été constamment en colère, mais nous ne pouvions pas comprendre clairement ses événements traumatiques car ses aptitudes de compréhension verbale étaient détériorées. Il a été conclu qu'il était hautement probable que le patient souffrait 
TABLEAU 4. Extraits du dossier du Cas 3 rédigés par les soignants

\begin{tabular}{|c|c|c|c|}
\hline Période & Jour/mois & Heure & État et comportements du patient \\
\hline \multirow{16}{*}{$\begin{array}{l}\text { Période d'évaluation } \\
\text { initiale }\end{array}$} & \multirow[t]{8}{*}{$9 / 5$} & $11 \mathrm{~h} 30$ & Se lève souvent. Excitation psychomotrice. \\
\hline & & $14 \mathrm{~h} 00$ & $\begin{array}{l}\text { Est assisté pour se laver. A besoin du soutien du soignant à } \\
\text { chaque étape du déshabillage et de l'habillage. }\end{array}$ \\
\hline & & $18 \mathrm{~h} 00$ & $\begin{array}{l}\text { Tend la main pour attraper la nourriture disponible. } \\
\text { Excitation psychomotrice. }\end{array}$ \\
\hline & & $19 \mathrm{~h} 00$ & Marche seul. Déambulation. \\
\hline & & $20 \mathrm{~h} 00$ & S’endort sur le canapé du couloir. Se réveille après 30 minutes. \\
\hline & & $22 \mathrm{~h} 00$ & Chante à tue-tête. \\
\hline & & $22 \mathrm{~h} 30$ & Reçoit ses médicaments en unidose. Mord le gobelet. \\
\hline & & $23 \mathrm{~h} 00$ & Chante à tue-tête. \\
\hline & \multirow[t]{6}{*}{$10 / 5$} & 0h30 & S’assied dans son lit. Se lève. Doit être surveillé de près. \\
\hline & & $1 \mathrm{~h} 30$ & Se tient debout sur son lit. Surveillé de près. \\
\hline & & 3h00 & S’assied dans son lit. Se lève parfois. Doit être surveillé. \\
\hline & & $6 \mathrm{~h} 00$ & Se met parfois debout sur son lit. Doit être surveillé. \\
\hline & & $7 \mathrm{~h} 40$ & $\begin{array}{l}\text { Petit déjeuner. Excitation psychomotrice. Essaie de prendre } \\
\text { les aliments des assiettes des autres résidents. }\end{array}$ \\
\hline & & En journée & $\begin{array}{l}\text { Hurle et se déplace sans cesse. Excitation psychomotrice. } \\
\text { Prise en charge individuelle. }\end{array}$ \\
\hline & \multirow[t]{2}{*}{$11 / 5$} & oh00 & En étant sur le lit, essaie d’en enlever les barrières. \\
\hline & & En journée & $\begin{array}{l}\text { Crie fort, se déplace sans cesse, excitation psychomotrice } \\
\text { toute la journée. Prise en charge individuelle. }\end{array}$ \\
\hline \multirow{8}{*}{$\begin{array}{l}\text { Période d'intervention I } \\
+ \text { traitement } \\
\text { pharmacologique }\end{array}$} & $16 / 5$ & & Début du traitement pharmacologique \\
\hline & \multirow[t]{4}{*}{$17 / 5$} & $10 \mathrm{~h} 30$ & Excitation psychomotrice. Se calme un peu. \\
\hline & & En journée & $\begin{array}{l}\text { Tape dans les mains. Se parle à lui-même. Se lève. Prise en } \\
\text { charge individuelle. }\end{array}$ \\
\hline & & $20 \mathrm{~h} 00$ & S'endort mais se lève rapidement de nouveau. \\
\hline & & $22 \mathrm{~h} 00$ & Se lève, ouvre la porte. \\
\hline & \multirow[t]{2}{*}{$18 / 5$} & oh00 & Se réveille. S’assied à côté du lit voisin. \\
\hline & & En journée & $\begin{array}{l}\text { Tape dans les mains. Se parle à lui-même. Se lève. Prise en } \\
\text { charge individuelle nécessaire. }\end{array}$ \\
\hline & $19 / 5$ & En journée & $\begin{array}{l}\text { Tape dans les mains. Se parle à lui-même. Se lève. Prise en } \\
\text { charge individuelle nécessaire. }\end{array}$ \\
\hline \multirow{8}{*}{$\begin{array}{l}\text { Période d'intervention II } \\
\quad+\text { traitement } \\
\text { pharmacologique }\end{array}$} & \multirow[t]{2}{*}{$25 / 5$} & $13 \mathrm{~h} 00$ & Séance 1 d'EMDR. \\
\hline & & En journée & $\begin{array}{l}\text { Tape dans les mains. Se parle à lui-même. Se lève. Prise en } \\
\text { charge individuelle, mais pas d'excitation psychomotrice. }\end{array}$ \\
\hline & \multirow[t]{5}{*}{$26 / 5$} & oh00 & Se réveille. \\
\hline & & $3 \mathrm{~h} 00$ & $\begin{array}{l}\text { Se parle à lui-même dans sa chambre. Se tient debout. } \\
\text { Insomnie. }\end{array}$ \\
\hline & & $13 \mathrm{~h} 00$ & Séance 2 d'EMDR. \\
\hline & & En journée & $\begin{array}{l}\text { Se calme. Pas d'excitation psychomotrice. Prise en charge } \\
\text { individuelle. }\end{array}$ \\
\hline & & $23 \mathrm{~h} 30$ & $\begin{array}{l}\text { Se tient debout, torse nu. Quand on l'encourage à dormir, } \\
\text { s'endort immédiatement. }\end{array}$ \\
\hline & $27 / 5$ & En journée & Est calme. Pas de prise en charge individuelle. \\
\hline
\end{tabular}

(Continued) 
TABLEAU 4. Extraits du dossier du Cas 3 rédigés par les soignants (Continued)

\begin{tabular}{|c|c|c|c|}
\hline Période & Jour/mois & Heure & État et comportements du patient \\
\hline \multirow[t]{14}{*}{$\begin{array}{l}\text { Période d'intervention III } \\
\quad+\text { traitement } \\
\text { pharmacologique }\end{array}$} & $30 / 5$ & $19 \mathrm{~h} 00$ & $\begin{array}{l}\text { Séance } 3 \text { d'EMDR (séance DIR). Était très actif depuis qu'il a } \\
\text { réintégré la maison. Une séance de DIR est réalisée en raison } \\
\text { de son état d'agitation. S'endort pendant la séance. }\end{array}$ \\
\hline & & En journée & Pas de prise en charge individuelle. \\
\hline & $31 / 5$ & oh00 & $\begin{array}{l}\text { Se réveille. Quand on l'encourage à dormir, } \\
\text { s'endort immédiatement. }\end{array}$ \\
\hline & & En journée & Pas de prise en charge individuelle. \\
\hline & $1 / 6$ & $2 \mathrm{~h} 00$ & $\begin{array}{l}\text { Se réveille. Se parle à lui-même. Est bavard, se déplace. } \\
\text { S'endort à } 3 \mathrm{~h} 30 .\end{array}$ \\
\hline & & En journée & Est calme. Pas de prise en charge individuelle. \\
\hline & $2 / 6$ & En journée & Est calme. Pas de prise en charge individuelle. \\
\hline & $6 / 6$ & En journée & Est calme. Pas de prise en charge individuelle. \\
\hline & & $20 \mathrm{~h} 00$ & Était très actif. S’endort paisiblement pendant la séance DIR. \\
\hline & $7 / 6$ & En journée & Est calme. Pas de prise en charge individuelle. \\
\hline & & La nuit & S'endort à $22 \mathrm{~h} 00$. Dort paisiblement. \\
\hline & $8 / 6$ & En journée & Est calme. Pas de prise en charge individuelle. \\
\hline & & La nuit & S’endort à $22 \mathrm{~h} 00$. Dort paisiblement. \\
\hline & $9 / 6$ & $13 \mathrm{~h} 00$ & $\begin{array}{l}\text { Séance } 5 \text { d'EMDR (séance DIR). Est actif. Séance réalisée par } \\
\text { le soignant. }\end{array}$ \\
\hline
\end{tabular}

Note. DIR = développement et installation de ressources.

de reviviscences d'événements traumatiques et que le traitement de souvenirs avec l'EMDR a conduit à une importante amélioration des SCPD. Toshio s'est calmé et souriait plus souvent après l'EMDR. Nous avons souvent utilisé le développement et installation de ressources (Korn \& Leeds, 2002) pour le calmer ; les tapotements bilatéraux non verbaux entraînaient un sentiment de sécurité chez ce patient adulte âgé atteint d'une démence sévère et constituent un moyen efficace pour apporter une stabilité à de tels patients. La stimulation bilatérale amoindrissait peut-être le stimulus auquel il réagissait. Ces effets se sont maintenus pendant plus de 6 mois.

\section{Discussion}

Nous avons utilisé l'EMDR-sur-le-champ avec trois patients atteints d'une démence modérée à sévère. Tomoko était une femme âgée de 67 ans ; à la suite de cette approche, son excitation psychomotrice et son agitation ont clairement diminué. Taro était un homme âgé de 85 ans; ses cris soudains ont disparu. Enfin, Toshio était un homme de 83 ans ; son excitation psychomotrice était gérée par les tapotements bilatéraux administrés par les soignants et la fréquence de ses éclats de colère s'est réduite. En considérant ces résultats, on peut affirmer que l'EMDR-sur-le-champ a conduit à une amélioration marquée des SCPD chez deux patients et a pu avoir une forte influence sur le soulagement des symptômes chez un troisième patient.

Comme ces trois patients manifestaient des symptômes cliniques de SCPD dans lesquels ils semblaient connaître et revivre des événements traumatiques de manière répétée, l'administration expérimentale de l'EMDR paraissait appropriée. Des modifications nécessaires ont été apportées aux procédures EMDR standard, résultant en la méthode EMDR-sur-lechamp destinée aux patients atteints d'une démence sévère.

Cette étude a investigué l'efficacité de cette méthode. Les symptômes SCPD étaient évalués à travers l'observation clinique car aucune autoévaluation n'était possible. Cette étude a montré que des résultats significatifs tels que l'élimination de plusieurs symptômes pouvaient s'obtenir avec l'EMDR-surle-champ. Bien sûr, comme les trois patients avaient une démence sévère, la déambulation fréquente et l'excitation psychomotrice n'ont pas disparu complètement, mais les agressions physiques soudaines, les cris et le langage violent ont considérablement diminué. 
Il semble qu'un retraitement de souvenirs a eu lieu, particulièrement pour les patients 1 et 2 , puisque le contenu des verbalisations et des comportements SCPD a changé. Avant l'EMDR-sur-le-champ, ces individus criaient, hurlaient et se comportaient d'une manière qui faisait penser aux traumas passés qu'ils avaient vécus. Après l'EMDR-sur-le-champ, les patients n'avaient plus de tels épisodes émotionnellement perturbants. On sait que pour les personnes atteintes de démence, un traitement efficace n'est généralement obtenu que pendant la période d'intervention de la psychothérapie. Dans ces cas, cependant, les effets du traitement EMDR-sur-le-champ étaient encore manifestes pendant la période de suivi de plus de 6 mois

Le développement et installation de ressources (Korn \& Leeds, 2002), qui développe et installe une cognition positive chez le patient, a été administré aux patients comme la thérapie par réminiscence, avec des stimulations des deux côtés du corps. On pense que ceci pourrait faciliter l'accès à des émotions et à des souvenirs positifs, stabilisant ainsi les patients atteints de démence souffrant de désorientation. Nous faisons l'hypothèse que la stimulation bilatérale a l'avantage d'aider les patients atteints de démence à se remémorer les souvenirs non seulement verbalement mais aussi à travers leurs perceptions sensorielles. Nous avons également observé que l'apport du développement et installation de ressources aux patients agités entraînait un effet apaisant.

\section{Mécanismes d'action possibles}

L'EMDR est une psychothérapie qui accède aux souvenirs passés et particulièrement aux souvenirs traumatiques (Shapiro, 2001). Un patient présentant des SCPD sévères et qui hurle est envisagé comme une personne qui revit un trauma à cet instant précis, comme un flash-back. L'EMDR est utilisé pour stimuler les deux côtés du corps pendant la reviviscence, dans l'attente que le souvenir traumatique pourra ainsi être retraité. L'EMDR standard est reconnu comme un traitement efficace de l'ESPT (p. ex. Bisson \& Andrew, 2007) et l'élimination des symptômes s'accompagne typiquement de changements au niveau de la perturbation émotionnelle liée au souvenir et des appréciations cognitives (p. ex. de " je suis en danger » à "c'est fini »). On a montré que la méthode EMDR-sur-le-champ diminuait la perturbation émotionnelle observée des trois patients ainsi que leurs verbalisations et comportements émotionnels liés aux traumas.
Puisque le but principal de cette étude était d'examiner l'efficacité de l'EMDR pour les SCPD chez des patients atteints de démence, il semble difficile de spéculer sur le mécanisme thérapeutique de l'EMDR à partir des résultats actuels. Certains pourront présumer que, puisque les patients avaient un fonctionnement cognitif faible, le mécanisme devait être les tapotements bilatéraux. Il existe en effet des preuves des effets thérapeutiques de la stimulation bilatérale (mouvements oculaires) utilisée en EMDR. Un large corpus de recherches a trouvé de nombreux effets, comprenant l'apaisement physiologique (p. ex. Sack, Lempa, Steinmetz, Lamprecht \& Hofmann, 2008), la désensibilisation des souvenirs perturbants (Van den Hout \& Engelhard, 2012) et l'amélioration du rappel des souvenirs (p. ex. Parker, Relph $\&$ Dagnall, 2008).

Toutefois, on ne s'attendrait pas à ce que les effets des tapotements bilatéraux perdurent au fil du temps, or nos trois patients ont connu des effets à long terme avec la méthode EMDR-sur-le-champ, avec des preuves indiquant que les souvenirs n'étaient plus perturbants. C'est pourquoi il apparaît qu'il y a un effet lié au retraitement de souvenirs. Ce changement est prédit par le modèle du traitement adaptatif de l'information de Shapiro (2001) qui postule que, lorsqu'on accède à un souvenir en EMDR, les procédures mènent à une reconsolidation ou à un changement du souvenir tel qu'il est conservé dans le cerveau (Solomon \& Shapiro, 2008). Nous prônons une future investigation de la méthode EMDR-sur-lechamp. La recherche pourrait comparer les effets d'un simple apport de tapotements bilatéraux aux effets de la méthode EMDR-sur-le-champ complète décrite dans cet article.

Il est aussi intéressant qu'important de noter que le traitement EMDR de nos patients atteints de démence était efficace malgré des déficiences cognitives considérables dues à des lésions cérébrales organiques qui se manifestaient en tant qu'atrophie pathologique dans le cerveau, particulièrement dans le cortex préfrontal et le cortex temporal. Bremner (2007) a déclaré que le lobe préfrontal joue un rôle important dans le traitement de l'ESPT et Pagani et coll. (2011) et Pagani et coll. (2012) ont soutenu cette hypothèse lors de séances EMDR dans une étude par électroencéphalogramme. Il semble cependant peu probable que le cortex préfrontal ait joué un rôle majeur dans le traitement des SCPD par l'EMDR dans nos cas. Une faible possibilité de l'implication du cortex préfrontal dans le mécanisme thérapeutique de l'EMDR était également suggérée par des études auprès de patients atteints de handicaps intellectuels et 
développementaux (HID). Les études déclarent que le traitement EMDR fut apporté avec succès aux patients atteints de HID (Barol \& Seubert, 2010 ; Mevissen et coll., 2011). Si le cortex préfrontal constitue une condition nécessaire pour les effets de l'EMDR, alors nos patients atteints de démence et les patients atteints de HID ne montreraient pas d'amélioration. Notre étude précédente par spectroscopie proche infrarouge indiquait que le flux sanguin augmentait pendant le rappel de souvenirs traumatiques et diminuait pendant les mouvements oculaires dans le sillon temporal supérieur droit sans changements significatifs dans le cortex préfrontal pendant les séances EMDR (Amano, Seiyama \& Toichi, 2010, 2012, 2013 ; Amano, Toyoda, Seiyama $\&$ Toichi, 2011). Des recherches supplémentaires sont nécessaires pour clarifier les mécanismes cérébraux qui jouent un rôle majeur dans le retraitement EMDR.

\section{Démence et ESPT}

Incidemment, les patients Taro et Toshio étaient des vétérans de la deuxième guerre mondiale. Yaffe et coll. (2010) ont effectué une recherche concernant "ESPT et risque de démence » dans la base de données nationale des soins des patients du ministère des anciens combattants [Department of Veterans Affairs National Patient Care Database] et ont trouvé que les personnes avec un diagnostic d'ESPT couraient deux fois plus de risque de développer une démence en comparaison des personnes non atteintes d'un ESPT. Yehuda et coll. (2005) ont mené une recherche auprès de rescapés de l'holocauste atteints d'ESPT et ont déclaré que l'ESPT pourrait accélérer le "processus de sénescence " de manière générale. Il existe plusieurs raisons pour lesquelles les patients atteints d'ESPT pourraient présenter un risque accru de développer une démence. L'une de celles-ci pourrait être le stress chronique qui endommagerait l'hippocampe (Schmitt et coll., 2004 ; Schuff et coll., 2001). Des volumes hippocampiques réduits ont été associés à un fonctionnement cognitif faible et à un risque accru de démence chez des personnes âgées en bonne santé (Grundman et coll., 2002). Il est possible que l'ESPT conduise à une atrophie hippocampique qui à son tour augmente le risque de déficits cognitifs et de démence (Yaffe et coll., 2010). Les symptômes de nos trois patients ont pu se développer ou avoir été accélérés par l'ESPT.

Nous proposons qu'en ce qui concerne les symptômes SCPD, il pourrait s'avérer intéressant d'envisager l'inclusion d'un syndrome clinique de type ESPT. Dans de tels cas, un traitement du trauma comme l'EMDR-sur-le-champ est nécessaire. Il faut noter que seulement quatre à six séances d'intervention ont été apportées par la psychothérapeute (premier auteur) et que le traitement était appuyé par les soignants qui utilisaient la stimulation bilatérale en réaffirmant que le trauma était fini. Cette intervention s'est avérée très efficiente et efficace, réduisant de manière notable la détresse émotionnelle éprouvée par ces patients.

\section{Résumé}

En conclusion, les patients atteints de SCPD possèdent souvent des caractéristiques similaires aux symptômes traumatiques en lien avec le rappel d'événements passés et des réactions émotionnelles associées. Dans cette étude, nous avons utilisé l'EMDR-sur-le-champ avec des patients atteints d'une démence sévère à un stade tardif pour la première fois, avec des effets thérapeutiques. Comme le protocole EMDR standard ne peut être directement appliqué aux patients déments, certaines modifications telles que celles de la méthode EMDR-sur-le-champ semblent nécessaires. À l'aide de ce protocole modifié, tous les cas ont manifesté une amélioration prononcée des SCPD qui s'est maintenue pendant la période de suivi de plus de six mois. Il est possible que, certains SCPD pouvant être associés à un syndrome de type ESPT, l'EMDR puisse être utilisé avec des patients atteints de démence et que l'EMDR-sur-le-champ puisse présenter un grand potentiel en tant que traitement non pharmacologique de certains SCPD. Aussi, les résultats de cette étude pourraient contribuer à la clarification des mécanismes neurobiologiques de l'EMDR.

\section{Note}

1. NdT : la personne suit et imite son proche ou son soignant à chaque instant - elle devient votre ombre.

\section{Bibliographie}

Amano, T., \& Ichii, M. (2009). Treatment of behavioral and psychological symptoms of dementia (BPSD) with EMDR. Japanese Journal of EMDR Research \& Practice, 1, 24-33.

Amano, T., Seiyama, A., \& Toichi, M. (2010). Phantom limb pain protocol of eye movement desensitization and re- processing (EMDR) for chronic pain: A case report. Journal of Japan Society of Pain Clinicians (JJSPC), 17(1), 29-33.

Amano, T., Seiyama, A., \& Toichi, M. (2012). Activation of the brain cortex measured by near-infrared spectroscopy 
during ego state therapy with EMDR in dissociative disorder. Japanese Journal of EMDR Research \& Practice, 4, $18-28$.

Amano, T., Seiyama, A., \& Toichi, T. (2013). The activity of the brain cortex measured by near-infrared spectroscopy (NIRS) during a session of phantom limb pain (PLP) protocol of eye movement desensitization and reprocessing (EMDR). Journal of EMDR Practice and Research, 7(3), 144-153.

Amano, T., Toyoda, M., Seiyama, A., \& Toichi, M. (2011). PTSD niyoru manseitu ni taisuru gankyuundo niyoru EMDR no chiryokatei de mirareta nouketuryu enka [Blood flow changes during a treatment session in a pa- tient with chronic pain due to posttraumatic stress disorder (PTSD)]. Journal of Japan Society of Pain Clinicians, 18(2), 55-57.

American Psychiatric Association. (1999). Practice guidelines for the treatment of psychiatric disorders: Compendium (1999). Washington, DC: Author.

Barol, B. I., \& Seubert, A. (2010). Stepping stones: EMDR treatment of individuals with intellectual and developmental disabilities and challenging behavior. Journal of EMDR Practice and Research, 4, 156-169.

Bisson, J., \& Andrew, M. (2007). Psychological treatment of post-traumatic stress disorder (PTSD). Cochrane Database of Systematic Reviews, (3), CD003388.

Bonder, B. R. (1994). Psychotherapy for individuals with Alzheimer disease. Alzheimer Disease \& Associated Disorders, 8(3), 75-81.

Bremner, J. D. (2007). Functional neuroimaging in post- traumatic stress disorder. Expert Review of Neurotherapeutics, 7, 393-405.

Butler, R. N. (1963). The life review: An interpretation of reminiscence in the aged. Psychiatry, 26, 65-76.

Cohen-Mansfield, J. (1986). Agitated behaviors in the elderly. Preliminary results in the cognitively deteriorated. Journal of American Geriatric Society, 34, 722-727.

Group for the Advancement of Psychiatry Committee on Aging. (1988). The psychiatric treatment of Alzheimer disease. Report 125. New York, NY: Brunner/Mazel.

Grundman, M., Sencakova, D., Jack, C. R., Jr., Petersen, R. C., Kim, H. T., Schultz, A., . . . Thal, L. J. (2002). Brain MRI hippocampal volume and prediction of clinical status in a mild cognitive impairment trial. Journal of Molecular Neuroscience, 19(1-2), 23-27.

Hyer, L. (1995). Use of EMDR in a "dementing" PTSD survivor. Clinical Gerontologist, 16(1), 70-73.

Jones, G. M. (1985). Validation therapy: A companion to reality orientation. The Canadian Nurse, 81(3), 20-24.

Kitwood, T. (1993). Person and process in dementia. International Journal of Geriatric Psychiatry, 8, 541-545.

Korn, D., \& Leeds, A. (2002). Preliminary evidence of efficacy for EMDR resource development and installation in the stabilization phase of treatment of complex posttraumatic stress disorder. Journal of Clinical Psychology, 58(12), 1465-1487.
Lavett, J. (1999). Small wonders: Healing childhood trauma with EMDR. New York, NY: The Free Press.

Lawlor, B. A. (2002). Managing behavioural and psychological symptoms in dementia. The British Journal of Psychiatry, 181, 463-465.

Mevissen, L., Lievegoed, R., Seubert, A., \& Jongh, A. D. (2011). Do persons with intellectual disability and limited verbal capacities respond to trauma treatment? Journal of Intellectual \& Developmental Disability, 36(4), 278-283.

Pagani, M., Di Lorenzo, G., Monaco, L., Niolu, C., Siracusano, A., Verardo, A. R., . . Ammaniti, M. (2011). Pretreatment, intratreatment, and posttreatment EEG imaging of EMDR: Methodology and preliminary results from a single case. Journal of EMDR Practice and Research, 5, 42-56.

Pagani, M., Di Lorenzo, G., Verardo, A. R., Nicolais, G., Monaco, L., Lauretti, G., . . . Siracusano, A. (2012). Neurobiological correlates of EMDR monitoring-An EEG study. PloS One, 7(9), e45753.

Parker, A., Relph, S., \& Dagnall, N. (2008). Effects of bilateral eye movements on the retrieval of item, associative, and contextual information. Neuropsychology, 22(1), 136-145.

Sack, M., Lempa, W., Steinmetz, A., Lamprecht, F., \& Hofmann, A. (2008). Alterations in autonomic tone during trauma exposure using eye movement desensitization and reprocessing (EMDR)-Results of a preliminary. Journal of Anxiety Disorders, 22(7), 1264-1271.

Schmitt, B., Bernhardt, T., Moeller, H. J., \& Heuser, I. (2004). Combination therapy in Alzheimer's disease: A review of current evidence. CNS Drugs, 18, 827-844.

Schuff, N., Neylan, T. C., Lenoci, M. A., Du, A. T., Weiss, D. S., Marmar, C. R., \& Weiner, M. W. (2001). Decreased hippocampal $\mathrm{N}$-acetylaspartate in the absence of atrophy in posttraumatic stress disorder. Biological Psychiatry, 50(12), 952-959.

Shapiro, F. (1989a). Efficacy of the eye movement desensitization procedure in the treatment of traumatic memories. Journal of Traumatic Stress, 2, 199-223.

Shapiro, F. (1989b). Eye movement desensitization: A new treatment for post-traumatic stress disorder. Journal of Behavior Therapy and Experimental Psychiatry, 20(3), 211-217.

Shapiro, F. (2001). Eye movement desensitization and reprocessing: Basic principles, protocols and procedures ( $2^{\text {nd }} \mathrm{ed}$.). New York, NY: The Guilford Press.

Solomon, R. M., \& Shapiro, F. (2008). EMDR and the adaptive information processing model: Potential mechanisms of change. Journal of EMDR Practice and Research, 2(4), 315-325.

Thomas, R., \& Gafner, G. (1993). PTSD in an elderly male: Treatment with eye movement desensitization and reprocessing (EMDR). Clinical Gerontologist, 14(2), 57-59.

Van den Hout, M. A., \& Engelhard, I. M. (2012). How does EMDR work? Journal of Experimental Psychopathology, 3(5), 724-738. 
Woods, B., Spector, A. E., Jones, C. A., Orrell, M., \& Davies, S. P. (2009). Reminiscence therapy for dementia. The Cochrane Database of Systematic Reviews, (2), CD001120.

Yaffe, K., Vittinghoff, E., Lindquist, K., Barnes, D., Covinsky, K. E., Neylan, T., . . . Marmar, C. (2010). Posttraumatic stress disorder and risk of dementia among US veterans. Archives of General Psychiatry, 67(6), 608-613.

Yehuda, R., Golier, J. A., Harvey, P. D., Stavitsky, K., Kaufman, S., Grossman, R. A., \& Tischler, L. (2005). Relationship between cortisol and age-related memory impairments in Holocaust survivors with PTSD. Psycho- neuroendocrinology, 30(7), 678-687.

Remerciements. Les auteurs aimeraient remercier M. Ichii pour sa suggestion des séances EMDR.

Merci d'adresser toute correspondance concernant cet article à Tamaki Amano, Yoshida Konoe-cho, Sakyo-ku, Kyoto 606-8501, Japon. Courriel : yiu60432@nifty.com 